\title{
On the Apparatus Used for Longitudinal Vibration Experiments in Textile Fibers over the Range of Frequency from Subsonic to Supersonic
}

\author{
By Kiyohisa Fujino, Hiromichi Kawai, Tsuneo Horino and Kihachiro Miyamoto \\ Faculty of Engineering, Kyoto University, Kyoto-City
}

\begin{abstract}
Apparatus available for measuring the dynamic mechanical properties of textile fibers over a range of frequency from subsonic to supersonic are described in the following article. As an example of the results of experiments with the apparatus, some findings on Nylon-6 fiber are presented briefly. From these findings it appears that the so-called "Relaxation spectrum" of Nylon- 6 belongs to the category of the so-called "Box type distribution", which is a special feature of textile fibers, at least, over a range of relaxation time corresponding to the range of frequency measured.
\end{abstract}

\section{Introduction}

According to recently developed phenomenological theories on linear viscoelasticity, the so-called "relaxation spectrum" or "retardation spectrum" is the most fundamental function to unify the representations of such visccelastic properties of materials ${ }^{(1)}$.

In principle. the spectra can be exactly related in mathematical structure with every phenomenolcgical functions. such as the stress relaxation function $\phi(t)$, the strain creep function $\zeta^{\prime}(\mathrm{t})$, the complex dynamic modulus function $\mathrm{E}^{*}(\omega)$ and the complex dynamic compliance function $\mathrm{J}^{*}(\omega)$. Therefore, the spectra can be determined from any one of these phenomenological functions obtained from experiments.

In practice, however, mathematical transformations between phenomenological functions, such as the creep function and the relaxation function, as well as the spectra, are very complicated, and thus, the relaxation spectrum should be determined from the relaxation func tion or the complex dynamic modulus function and the retardation spectrum determined from the others, while the spectra are equivalent in the representation of mechanical properties of material to be tested.

But it is very difficult to determine a complete spectrum from eithery of these two phenomenological iunctions classified into the respective groups without any apprcximate. This is because an experiment to obtain the phencmenological function cannot be conducted over an exceedingly wide range of "external time scale," and also because, even if this cculd be done, the function cculdnot always be given as an analytical function.

Accordingly, the spectrum-for example, the relaxation spectrum - can be obtained only from stress relaxation experment in combination with vibration experiment using some approximate methods, where the former is suitable for long time scale experiment and the latter for short-time one.

This artiale briefly describes the apparatus available for dynamic experiments to determine the ccmplex dynamic modulus functions $\mathrm{E}^{*}(\omega)$ of textile fibers over a range of frequency frcm subsonic to supersonic, and gives only scme of the results cbtained by individual apparatus, since they are fully reported in another jcurnal ${ }^{(2)}$

\section{About the Apparatus Used}

To build the appartus to be used for determining the complex dynamic mcdulus function of textile fibers, it is necessary not only to suit the apparatus for vibration experiments cver the widest possible frequency range, but also to take into account the shape of textile fibers, their nature and the deformaticn mode adopted.

A single apparatus, however, cannot be used for such a widerange experiment. Several types of apparatus must be built for the respective frequency range experiments.

In all the experimental methcds employed here, the vibrational strain was always limited to pure longitudinal mcde only, and was kept smaller than $0.2 \%$ to avoid the non-linear characteristics. But it is well-known that on account of the special shape of textile fibers, that is to say, the length of a specimen, is very great compared with its crcss sectional dimension, compressicnal deformation is not realizable. Consequently, in order to avoid this drawback, as in several individual experimental prccedures which will be menticned later in this article, the initial static strain should be kept always 
larger than the vibrational strain by the application of a definite static load upon the sample strips.

But, strictly speaking, this initial static condition depends upon the actual construction of the individual apparatus and on how it is used. For example, the sample strip must be fixed lengthwise during an experiment with one apparatus, and this relaxes the initial definite static tension gradually. On the other hand, a constant tension is used with another arparatus, and so a static creep is unavoidably caused by the static tension. In other words, the former is a vibrational experiment under stress relaxation and the latter under creep of strain.

The magnitude of the dynamic strain amplitude itself was always kept smaller than $0.2 \%$, as mentioned above. but the actual magnitude varied in the opposite direction to the frequency range of the apparatus.

As mentioned in the introduction, to determine the relaxation spectrum from dynamic measurements, it is necessary to describe the experimental results by the complex dynamic mcdulus $E^{*}(\omega)$ as a function of angular frequency (") over the widest possible range. For linear viscoelastic substances, the complex dynamic modulus is defined by the ratio of complex amplitude of sinusoidal stress to that of strain. If the mechanical behavior under a given angular frequency is described by a single retarded elastic model, that is to say, a single Voigt element, the following simple mathematical relations can easily be obtained:

and

$$
\begin{aligned}
& E_{1}(\omega) \equiv E_{\omega} \\
& E_{\Theta}(\omega) \equiv \xi_{\omega} \cdot \omega
\end{aligned}
$$

$$
\tan \partial(\omega)=E_{9}(\omega) / E_{1}(\omega),
$$

where $E_{1}(\omega)$ and $E_{2}(\omega)$ are real and imaginary parts of complex dynamic modulus respectively ; $E$ and $\xi$ the tensile modulus of elasticity and the coefficient of normal viscosity, respectively, in a single Voigt element, and $\tan \delta$ the so-called mechanical loss tangens, inwhich $\delta$ is the phase difference between stress and strain amplitudes.

Our experimental objectve was to determine $E_{1}(\omega)$ and $E_{2}(\omega)$ as a function of applied angular frequeney over the widestp ossible range several suitable experimental methods. The theory given later as to each type of measurement has been derived on the assump tion that the material tested behaved like a single Voigt element. The experimental results showed, in fact, that $E_{1}(\omega)$ and $E_{2}(\omega) / \omega$ were not independent of frequency, as had been assumed. However, it can be shown that even when the viscoelastic properties must be represented by a spectrum, the above assumption does, in fact, lead to the correct values of $E_{1}(\omega)$ and $E_{2}(\omega)^{(3)}$.

Because the deformation of the sample strip was simple tensile mode, and because the dimensions of a single textile fiber were tco small for accurate experimentation, the sample strips were always prepared in a bundle of single fibers by detwisting ordinary commercial multiple filaments yarn of about 100 den. Sample strips were pre-conditioned under standard air conditions, $20^{\circ} \mathrm{C}, 65 \%$ R. H., for several days and the experiments were also carried out under the same air conditions.

\section{Individual Apparatus and its Experimental Procedure}

(1) Apparatus used in the subsonic range.

The measurements over this frequency range were carried out by the free damping vibration methed, and the frequency range covered $2 \times 10^{-1}$ to $2 \times 10^{1} \mathrm{cps}$.

The construction of the apparatus used is illustrated in Fig. 1, where $D$ is a circular disc which has a known moment of inertia referring to its rotational axis. The circular disc is also fixed tightly by two chucks $A$ to a piece of piano wire $F$, which is vertically stretched by an adjustable screw I between two chucks $\mathrm{A}^{\prime}$, of which the lower one is fixed to the foundation and the upper one to the upper platform through the adjustable screw I. B are sample strips horizontally stretched by a definite dead weight
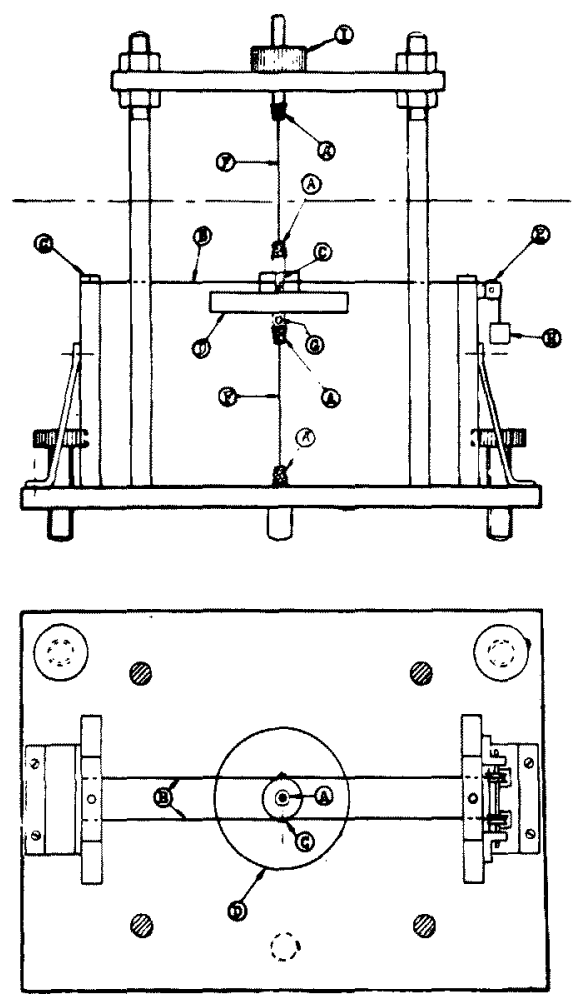

Fig. 1 
$\mathrm{H}$ through the idler pulleys $\mathrm{E}$. The sample strips are fixed at each end and in the center of their effective lengths by the sample clamps $\mathrm{C}$ to the foundations and to the circular disc. $\mathrm{G}$ is a 'small plane mirror cemented on the circular disc. Consequently, this forms a combined oscillation system in which the torsional oscillation system consisting of a circular disc and a piano wire is restrained by tensile alternating deformation of parallel sample strips.

Fig. 2 is a schematic diagram showing the general arrangement of the apparatus. The torsional free damping oscillation of the circular disc is started by mechanical exciter from a definite angular displacement. This oscillation is recorded together with a time signal generated from the tuning fork on the film drum by the aid of an optical system consisting of the two plane mirrors and the optical box of a Y.E.W. N-3 type electro-magnetic oscillograph as illustrated in the figure.

Fig. 3 is one of the typical records obtained by this apparatus. On analyzing the amplitude dependences both of the logarithmic decrement of the amplitude and of the period of oscillation, it is found that there are hardly any striking amplitude dependences in them, except a slight decrease in logarithmic decrement with decreasing amplitude; this is, however, somethat

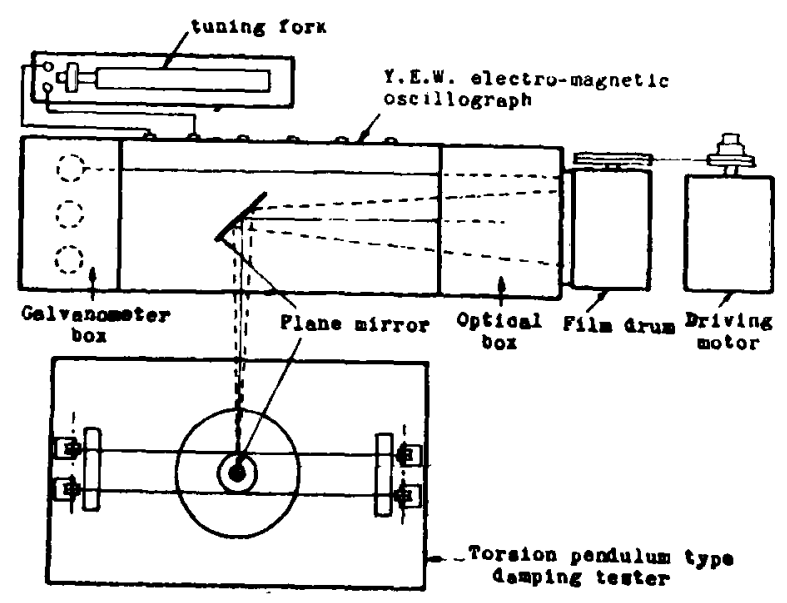

Fig. 2 marked in regenerated cellulose fibers.

Then, considering the non-linear effects, the fundamental equation of angular motion of the system referring to the axis of rotation may be given as follows: ${ }^{(4)}$

$$
a \ddot{\theta}+\underset{i}{\sum} \Sigma_{j} \beta_{i, j} \dot{\theta}|\dot{\theta}|^{p_{i}}|\theta|^{q_{j}}+\sum_{k} \gamma_{k} \theta|\theta|^{r_{k}}=0 \text {. }
$$

In approximate representation by which the damped oscillation just mentioned is reduced to a linear one with non-linear effects disregarded, Eq. (2) may be rewritten as follows:

$$
\alpha \ddot{\theta}+\beta \dot{\theta}+\lambda \theta=0 \text {. }
$$

Since the angular displacement $\theta$ is always kept very small and since the damping effects caused by internal friction of the piano wire and by air friction resulting from the rotation, are negligibly small, Eq. $\left(2^{\prime}\right)$ may be rewritten as follows by substituting the actual dimensions of the apparatus and the sample strips:

$$
I \ddot{\theta}+\left(\xi \frac{4 S}{l} a^{2}\right) \dot{\theta}+\left(E \frac{4 S}{l} a^{2}+b\right) \theta=0,
$$

where $E$ is the tensile modulus of elasticity of the sample strip, $\xi$ its coefficient of normal viscosity, 1 its effective length between the clamps, $S$ its effective cross sectional area, $I$ the moment of inertia of the circular disk referring to its axis, a the distance between the rotational axis and the sample clamp, and $b$ the torsional constant of the piano wire.

From the solution of Eq. (3) under a condition of free damped oscillation, the following mathematical expressions for $E$ and $\xi$ can easily be obtained:

$$
\begin{aligned}
E & =\frac{l I}{S a^{2}}\left\{\frac{\pi^{2}+(\ln \Delta)^{2}}{T^{2}}-\frac{\pi^{2}}{T_{0}^{2}}\right\} . \\
\xi & =\frac{l I}{S a^{2}} \frac{\ln \Delta}{T} . \quad \ldots \ldots \ldots \ldots \ldots \ldots \ldots \ldots \ldots \ldots
\end{aligned}
$$$$
\text { and }
$$

where $T$ and $T_{0}$ are oscillating periods of the system containing the sample strips and of the system not contaning them, respectively; and $\ln \Delta$ is the logarithmic decrement of the amplitudes in damped oscillation. Thus the two

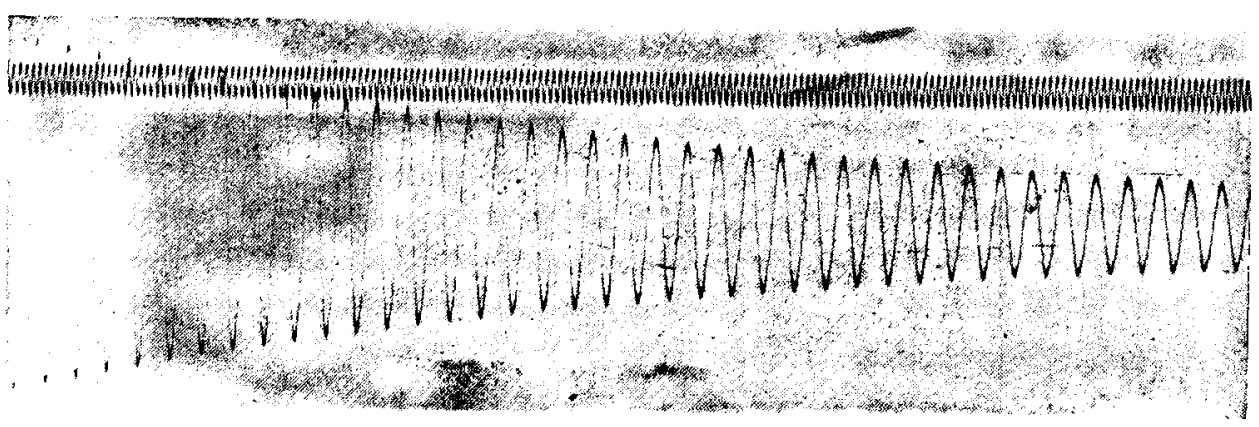

Fig. 3 One of the typical records of damped oscillation obtained by the apparatus (1) for Nylon-6 filaments of about 100 den. The timing marks are produced by 50 cps tuning fork. 


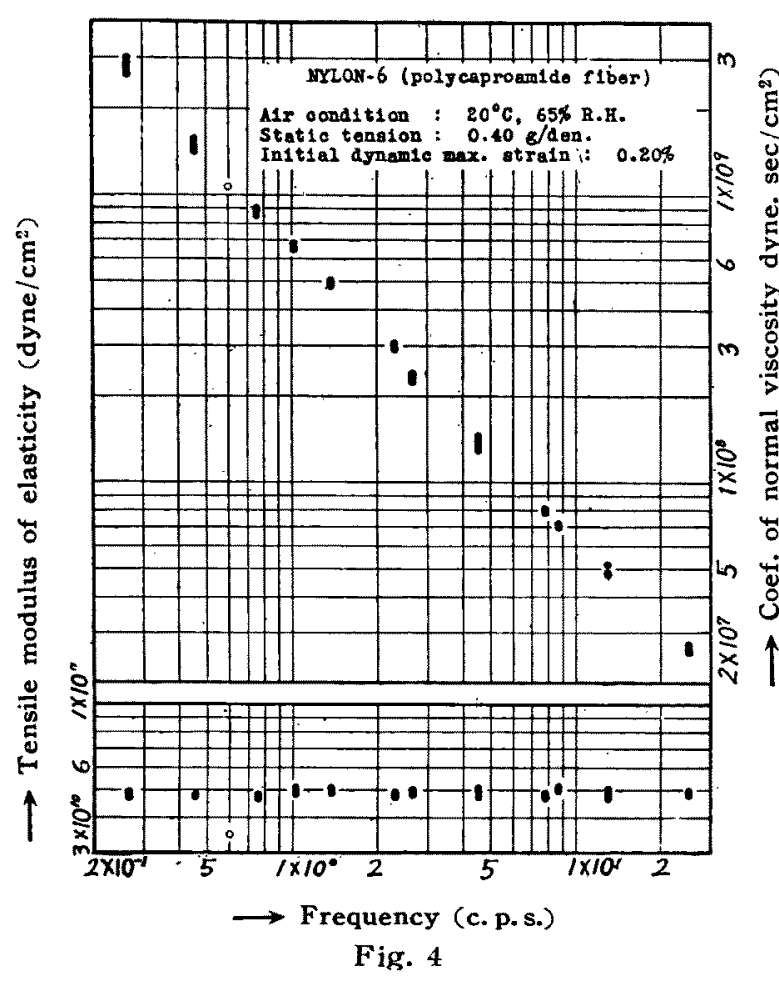

mechanical parameters $E$ and $\xi$, can be determined from the measurable quantities $T, T_{0}$ and $\ln s$.

As regards the practical procedures in this experiment, the maximum angular displacement of the circular disc was always kept at 2 degrees which was equivalent to about $0.2 \%$ longitudinal dynamic strain of the sample strip. The initial static tension of the sample strip was always kept at a definite value, about 0.4 $\mathrm{g} / \mathrm{den}$, by applying a dead weight to the sample strip; and changing of the angular frequency of system was carried out by changing the moment of inertia of circular disc from $3 \times 10^{2}$ to $3 \times 10^{5} \mathrm{cgs}$, or by changing the diameter of piano wire from 0.3 to $1.5 \mathrm{~mm}$, instead of changing the shape factor of the sample strip.

Fig. 4 is one of the results obtained by the apparatus for Nylon-6 filaments.

(2) Apparatus used in the low audio-range.

In order to extend the frequency range of experiment, the forced longitudinal vibration method was adopted, and thus the frequency range could be increased up to about $5 \times 10^{\circ} \mathrm{cps}$.

Because the apparatus and the principle employed here are essentially the same as those adopted by S. D. Gehman et al. for various gum stoks, ${ }^{(5)}$ by W. J. Lyons et al. for the strips of textiles in the so called "stretch vibrometer", ${ }^{(6)}$ by B. A. Dunell et al. for a single filament, ${ }^{(i)}$ and by G. Palandri for various tire cords, ${ }^{(8)}$ it may be unnecessary to describe the details except some modifications.

Fig. 5 is a schematic diagram of a ap-

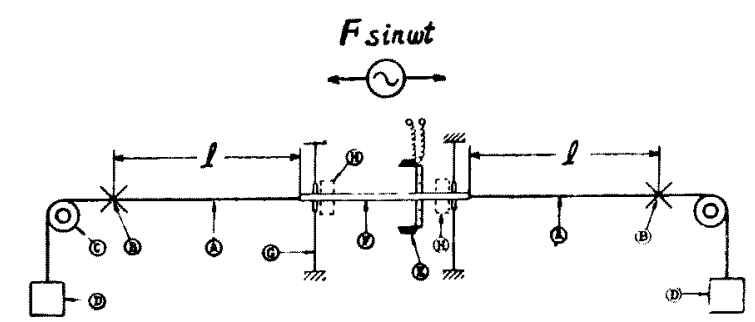

Fig. 5

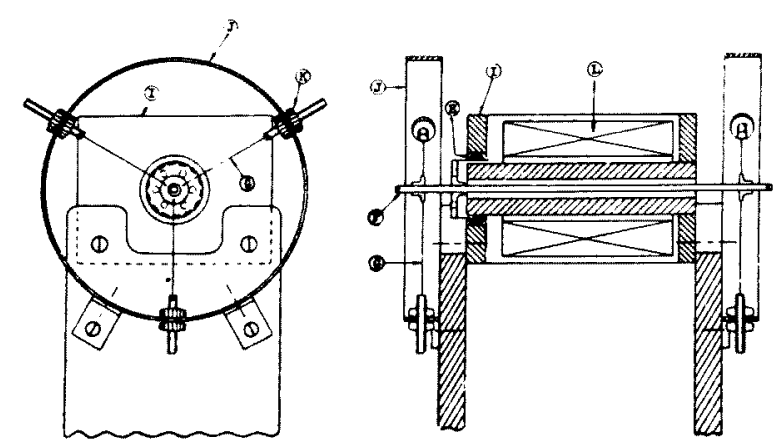

Fig. 6

paratus. Fig. 6 is a drawing of its driving part rebuilt from loud-speaker unit, where $A$ are sample strips stretched by a definite dead weight $\mathrm{D}$ through the idler pulleys $\mathrm{C}$. The sample strips are also connected with both ends of a horizontally movable light spindle $F$, and can be clamped at any point along their length by means of the movable clamps $\mathrm{B}$. $\mathrm{H}$ is a removable mass, loading the whole vibrator unit from about 8 to $50 \mathrm{gr}$. $\mathrm{E}$ is a solenoid coil cemented on the spindle $F$, and is located in the radial magnetic field which traverses an annular space between both poles of a magnetic core $I$, and $L$ is the exciting coil of this magnetic core. The horizontal movable spindle F is hung at each end and alined by the aid of fine nylon threads of about 15 den., which are radially stretched by the adjustable screws $\mathrm{K}$ mounted on the ring $\mathrm{J}$.

When direct and alternating currets are imposed on the exciting coil $\mathrm{L}$ and the driving coil $\mathrm{E}$, the longitudinal forced vibration of the system is excited.

Fig. 7 shows the general arrangement of the whole apparatus. The above mentioned vibrating system is built on a lathe-bed and located across the optical system which measures the amplitude of spindle by the photo-electric methcd. Since the driving of vibration of the spindle $\mathrm{F}$ is effected by supplying the alternating current from the audio frequency pcwer amplifier to the solenoid coil, the amplitude of this driving force can be determined by measuring the current through this coil, ramely, by measuring the voltage difference between the ends of a pure resistance coupled in series with the solenoid coil. 


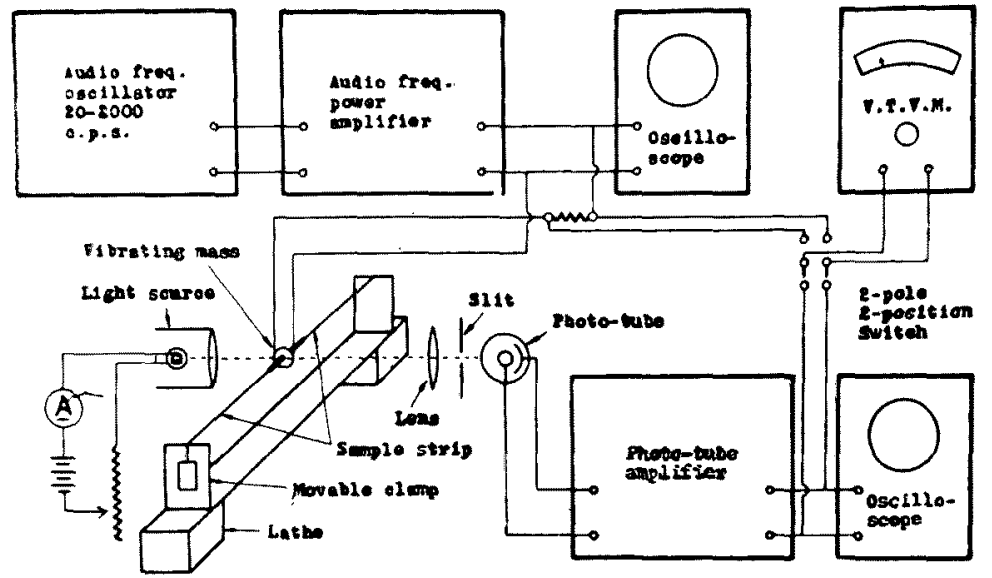

Fig. 7

The mechanical behavior of the samle strips under a small harmonic driving strain may well be represented by a single Voigt element. Again, when the amplitude of the vibrator itself is kept relatively small, there is hardly any noticeable non-linear behavior, which is presumably attributable to the construction of the vibrator assembly and results in an appreciable alteration of the resonance curve when a greater driving force is applied. Hence, the fundamental equation of motion of the vibrator under a small harmonic driving force, $F \sin \omega t$, can be approximated as follows:

$\alpha \ddot{x}+\beta \dot{x}+\gamma x=F \sin \omega t$,

where $x$ is displacement of the vibrator at time $t$.

By substituting the resonance conditions of the system into the steady state solution of Eq. (5), the final mathematical relations between two coefficients, $E$ and $\xi$, and measurable quantities are obtained under some adequate assumptions and ignoring of the influences of vibrator suspensions as follows:

$$
2 S E / l=M \omega_{i,}^{*}
$$

and

$$
2 S \xi / l=F /\left(A_{\max }\left(\omega_{0}\right),\right.
$$

where $M$ is effective mass of the vibrator and $A_{\max }$ the maximum amplitude of the vibrator at resonance angular frequency $\boldsymbol{\omega}_{0}$.

Thus, the two mechanical parameters $E$ and $\xi$ can be determined from the measurable quantities, $\omega_{0}, F$ and $A_{\max }$, at the resonance of the system. It seems necessary, however, to check the validity of neglecting non-linear forces caused by the suspensions, especially in experiments at relatively large vibration amplitude.

In practice, the experimental conditions were kept as nearly the same as the previous ones: the longitudinal dynamic strain and the initial static tension were always kept at definite values, $0.1 \%$ and about $0.4 \mathrm{~g} / \mathrm{den}$., respectively; and the experimental frequency, i.e., a resonance frequency of the system, was changed by altering the effective mass of the system from 8 to $50 \mathrm{gr}$. and by changing either the length of strips from 5 to $25 \mathrm{~cm}$ or the number of strips from one to several. Thus, the frequency range could cover from $5 \times 10$ to $5 \times 10^{2} \mathrm{cps}$.

Fig. 8 shows the result obtained by the apparatus for Nylon-6 filaments.

(3) Apparatus used in the frequency range from higher audio to supersonic.

By means of the longitudinal forced vibration method just mentioned, it was, in practice, difficult to experiment at higher frequencies than several hundred cycles per second, on account of the various restrictions, such as the effective mass of the system, the shape factor of sample strips and some disturbances of vibration. It was therefore necessary once again to change the experimental method to reach a higher frequency range.

The experimental frequency range was expanded by means of the longitudinal wave resonance method mainly in the higher audio frequency range, and by means of the longitudinal wave propagation method mainly in the

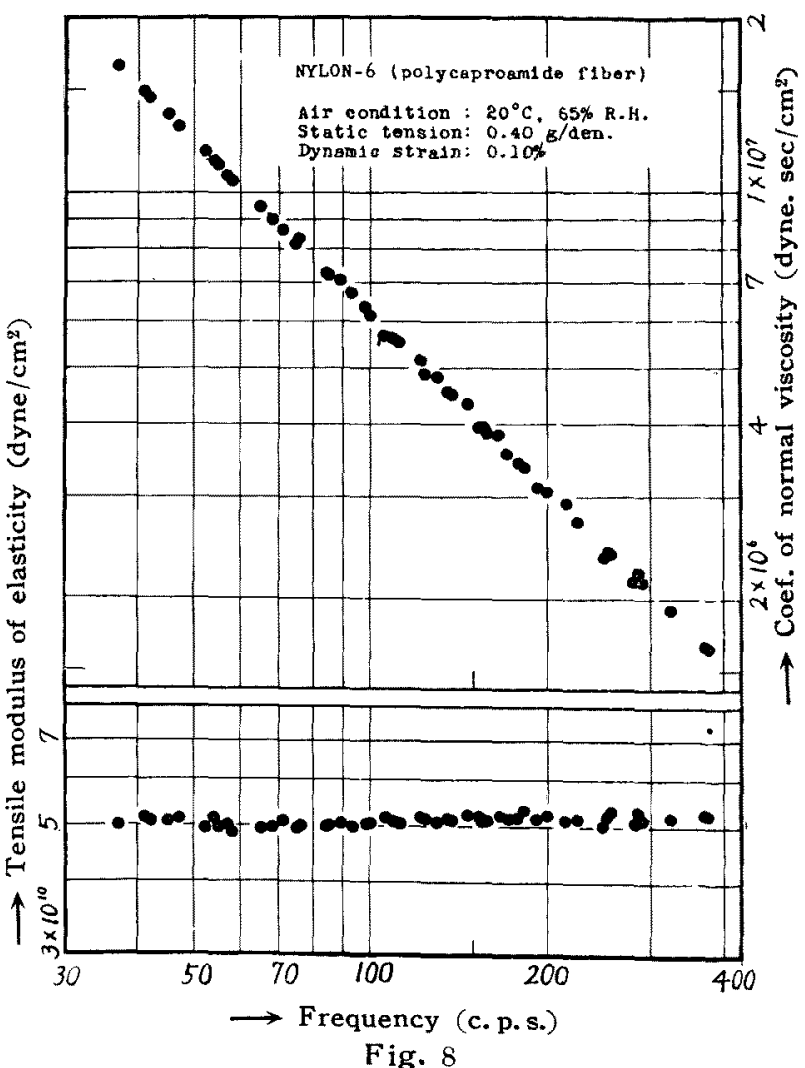

Fig. 8 
supersonic range, respectively; consequently the frequency range could cover from $2 \times 10^{3}$ to $2 \times 10^{5} \mathrm{cps}$.

Although the principles employed here are essentially the same as those advanced by A. W. Nolle ${ }^{(9)}$ in 1948, the apparatus is of a vertical type similar to that used by $\mathrm{H}$. Kawai and N. Tokita ${ }^{(10)(11)}$ and different from that of the horizontal one used by J. W. Ballou and S. Silverman et al. ${ }^{(9)(12)(17)}$ since 1944 for the propagation method. The conventional one has an unavoidable defect in that a contact condition between sample strip and wave detector is hardly kept constant during observation and consequently high accuracy of determination of attenuation constant cannot be expected.

Fig. 9 is the general arrangement of apparatus, where CR. 1 and CR. 2 are the driving and receiving crystals, respectively, which are cut out from a single crystal of Rochelle salt to be available for longitudinal vibration experiments. The sample strip is connected at one end with the driving crystal and at the other end with the vertically movable vernier scale, which is used for changing the effective length of the sample strip, through an idler pulley and a hook cemented on the receiving crystal. The driving crystal is not only driven by the output from the variable frequency signal generator but is also used as a dead weight together with the additional weight $W$. The cathode ray oscilloscope is used to measure the phase difference between the voltages on the sending and receiv. ing crystals to determine the wave length. Again, the two vacuum tube voltmeters, V.T. V.M. 1 and V.T.V.M. 2, are also used to measure the relative values of voltage amplitudes on sending and receiving crystals.

Therefore, this apparatus can be used for

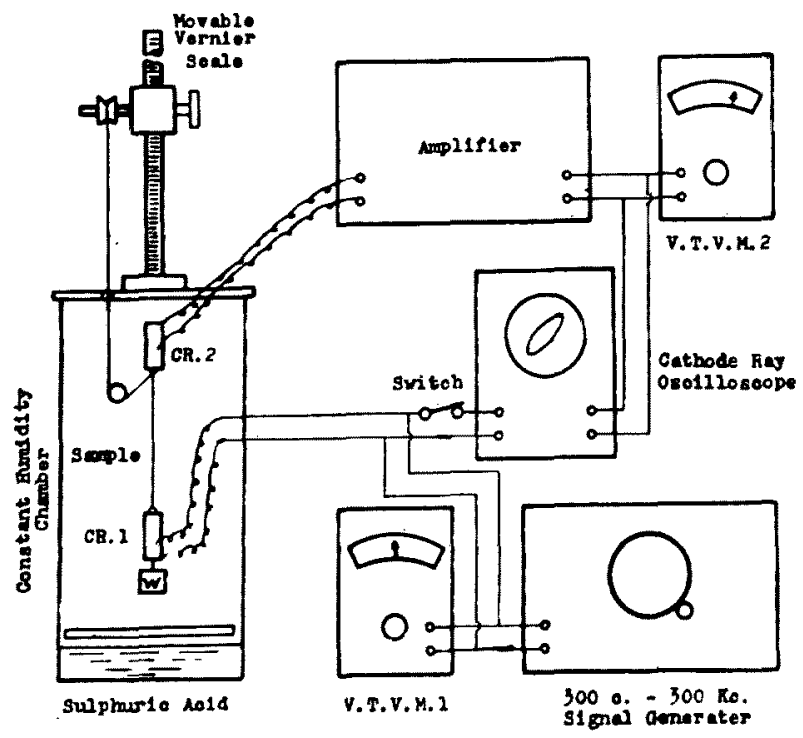

Fig. 9 both the abovementioned methcds. For the longitudinal wave resorance methcd the socalled resonance curve is obtained of the output voltage from CR. 2 against the driving frequency on CR. 1 while keeping the length of the sample strip constant. For the longitudinal wave propagation method the cutput voltage on CR. 2 is measured while keeping the driving frequency and voltage on $\mathrm{CR}$. 1 constant

Assuming here again that the non-restored force is proportional to the rate of change of strain and that the restored force is proportional to the strain itself, the following well-known wave equation emerges:

$$
\rho \frac{\partial^{2} \mu}{\partial t^{2}}=E \frac{\partial^{2} \mu}{\partial x^{2}}+\xi \frac{\partial^{3} \mu}{\partial x^{2} \partial t} .
$$

where $\mu$ is the instantaneous displacement of a cross section of the sample strip in the $x$ direction, $E$ the tensile modulus of elasticity, $\xi$ the coefficient of normal visccsity, and $\rho$ the density of the material.

Assuming that the driving force on the sample is sinusoidal one of angular frequency (1), then the steady state solution of Eq. (8) is given by the well-known equation as follows:

$$
l=\left(A_{1} e^{r x+i d r}+A_{2} e^{-(r r+i d r)}\right) e^{i \omega t},
$$

where $A_{1}$ and $A_{2}$ are constants which must be determined from the initial and boundary conditions of the experiments; $c$ and $d$ are attenuation constant and factor of phase shift, respectively, and they are represented as follows:

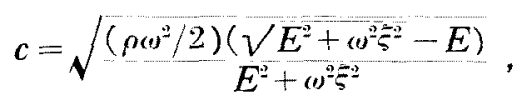

and

$$
d=\sqrt{\frac{\left(\rho \omega^{2} / 2\right)\left(\sqrt{E^{2}+\omega^{2} \xi^{2}}+E\right)}{E^{2}+\omega^{2} \xi^{2}}} .
$$

Furthermore, the two mechanical parameters of the sample strip, $E$ and $\xi$, are intrcduced from Eqs. (10) and (11) as follows:

$$
\begin{aligned}
& E=\rho v^{2}\left(\omega^{2}\left(\omega^{2}-c^{2} v^{2}\right) /\left(\omega^{2}+c^{2} v^{2}\right)^{2},\right. \\
& \text { and } \\
& \xi=2 \rho c v(\omega v)^{2} /\left(\omega^{2}+c^{2} v^{2}\right)^{2} . \ldots \ldots \ldots
\end{aligned}
$$

where $v$ is propagation wave velocity, and is equal to $\omega / d$.

The final equations, which are available for the determination of propagation constants, can be intrcduced by putting suitable experimental conditions into Eq. (9) as essentially the same as was done by A. W. Nolle ${ }^{(9)}$ for the longitudinal wave resonance methcd and by J. W. Ballou and J. C. Smith ${ }^{(1)}$ and K. W. Hillier and $\mathrm{H}$. Kolsky ${ }^{(15)}$ for the longitudinal wave profagation method.

Namely, for the resorance methcd under a 
fundamental longitudinal resonance mode of vibration;

and

$$
c \simeq\left(\begin{array}{l}
\pi \\
l
\end{array}\right) \quad \begin{array}{ll}
J_{\nu} & \frac{1}{2}
\end{array}
$$

$$
d=\frac{\pi}{l},
$$

where $l$ is the effective length of the sample strip and is equivalent to half the wave length $\lambda / 2 ; J_{2}$ is the so-called band width, the difference between two particular frequencies where the output voltage decreases to $1 / \sqrt{2}$ times more than that at resonance frequency $\nu_{r}$.

Fig. 10 shows resonance curves obtained by this method for Nylon-6. From the figure, the propagation constants mentioned above can be determined by using Eqs. (14) and (15).

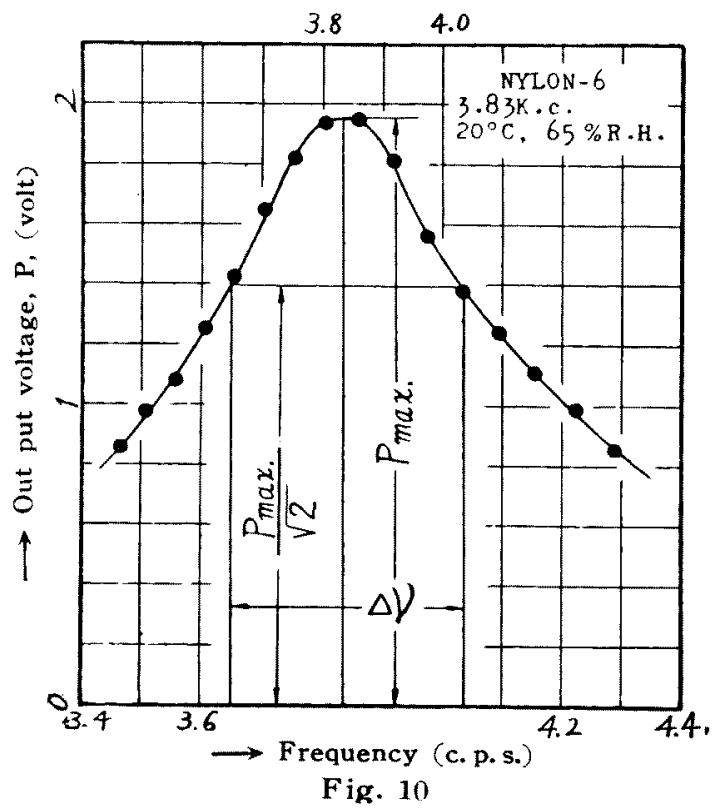

Furthermore, for the propagation method;

$$
\tan \theta=\frac{1+R \boldsymbol{e}^{-\underline{\Omega} r l}}{1-\boldsymbol{R} \boldsymbol{e}^{-\vartheta c l}} \tan (2 \pi l / \lambda),
$$

where $\theta$ is the phase difference between sending and receiving waves; $R$ the reflection coefficient at the receiver; and this equation is valid to determine $d$ from the diagram experimentally obtained between $\theta$ and $l$ by observing Lissajous patterns repeated on the screen of the cathod ray oscilloscope, and

$$
P(l)=K_{\left[1-2 R e^{--c l} \cos (\phi-4 \pi l / \lambda)+R^{2} e^{-4 c l}\right]^{1 / 2}},
$$

where $P(l)$ is reading of the output voltmeter, V.T.V.M. $2 ; K$ a constant; $e^{i \phi}$ refractive phase shift at the receiver; and this equation is available for the determination of $c$ from the diagram experimentally obtained between $P(l)$ and $l$.

If the attenuation constant $c$ is relatively large, namely the denominator of the right-hand side of the equation may be approximately equivalent to 1 , then the equation is rewritten as follows;

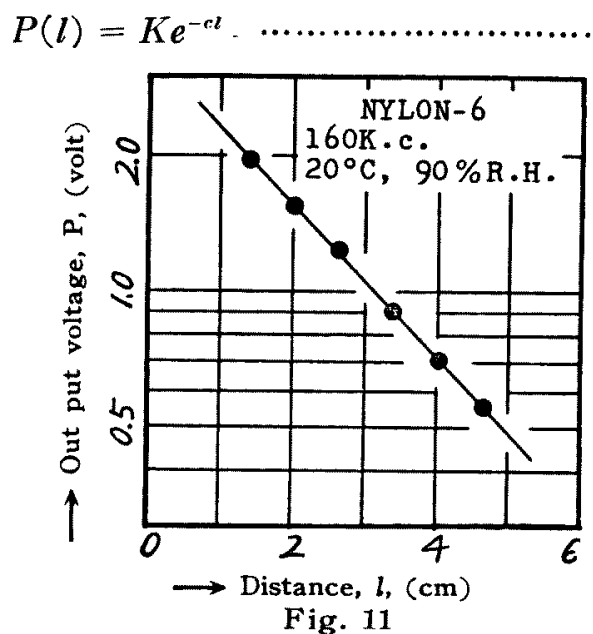

Fig. 11 is one of the examples in which Eq. (18) is valid to determine $c$ from the inclination of straight lineship between $\log P(l)$ and $l$.

If $c$ is not so large, then Eq. (17) is rewritten again as follows;

$$
P(l)=\frac{K^{\prime}}{\left[(1 / R) e^{2 c l}-2 \cos (\phi-4 \pi l / \lambda)+R e^{-2 e l}\right]^{1 / 2}} .
$$

$P(l)$ is demonstrated against $l$ by a damped curve having maxima and minima alternately at every points being at distance of $\lambda / 4$. Fig. 12 is the example for this case.

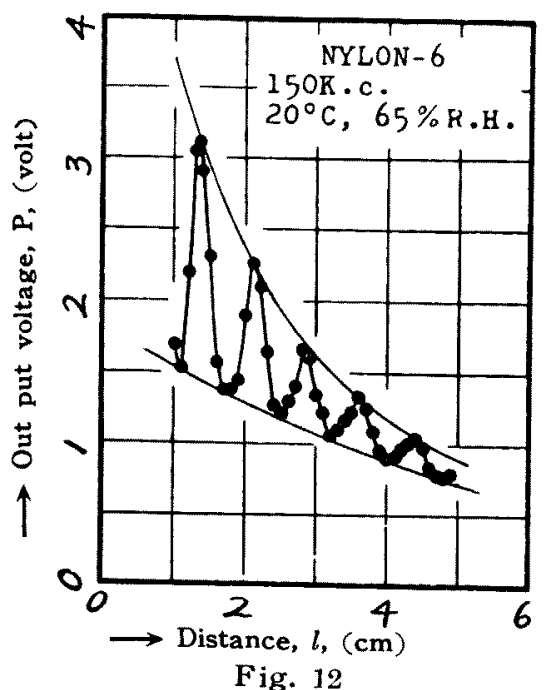

The equations of the envelop for the damped curve are obtained by purring $\cos (\phi-4 \pi l / \lambda)$ $= \pm 1$ and $R=e^{-k}$ into Eq. (19) as follows;

and

$$
E_{n v e l . \max }=K^{\prime} / 2 \sin h\left(c l+\frac{k}{2}\right)
$$

$$
E_{n v e l . . . \text { naln }}=K^{\prime} / 2 \cos h\left(c l+\frac{k}{2}\right),
$$




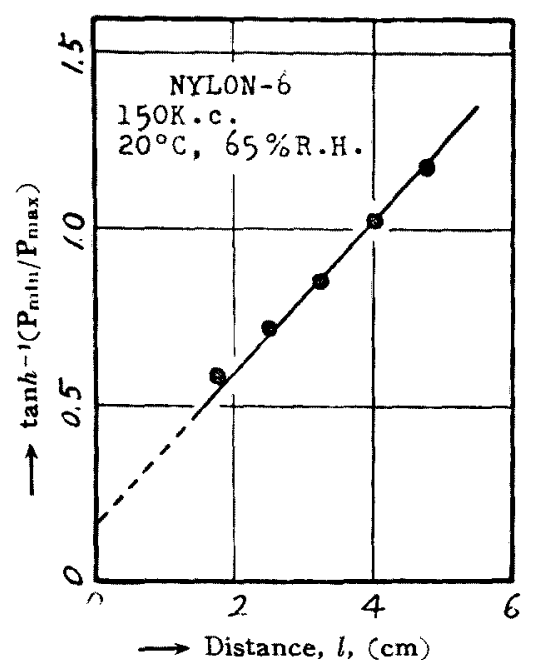

Fig. 13

then

$\tan h^{-1}\left(E_{n r e l \cdot \min } / E_{\text {nvel. nax }}\right)=c l+\frac{k}{2} \cdots(22)$

Fig. 13 shows the validity of Eq. (22) for the result metioned above. The attenuation constant $c$ can be determined from the inclination of the straight line.

Both the upper and lower limits of the frequency range covered by these experimental methods are determined by the acoustical shape of the sample strip, theoretically and by the dimension of the apparatus practically. The upper limit in our experiments, however, was in practice about $200 \mathrm{kc}$, which was appreciably lower than the theoretical one, on account of some experimental difficulties. On the other hand, the lower limit was about $40 \mathrm{kc}$ for the propagation method and about $2 \mathrm{kc}$ for the resonance one, being limited by the effective height of the closed vessel in which the sample strip was kept. Hence, experiments in the higher frequency range were carried cut mainly by the former method, while experements in the lower frequency range were carried out mainly by the latter.

The static tension of the sample strip was as nealry the same as in the previous experiments as possible. However, this was a dynamic experiment under creep and the former was under stress relaxation caused by clamping the sample during vibration. The dynamic strain amplitudes were extremely small compared with those in the previous experiments. These differences in the experimental conditions should be taken into account in comparing the results with thore obtained by the different methods.

Fig. 14 gives the results obtained by the apparatus for the attenuation constants of Nylon- 6 over a range of relative humidity from $5 \%$ to $50 \%$ R.H.

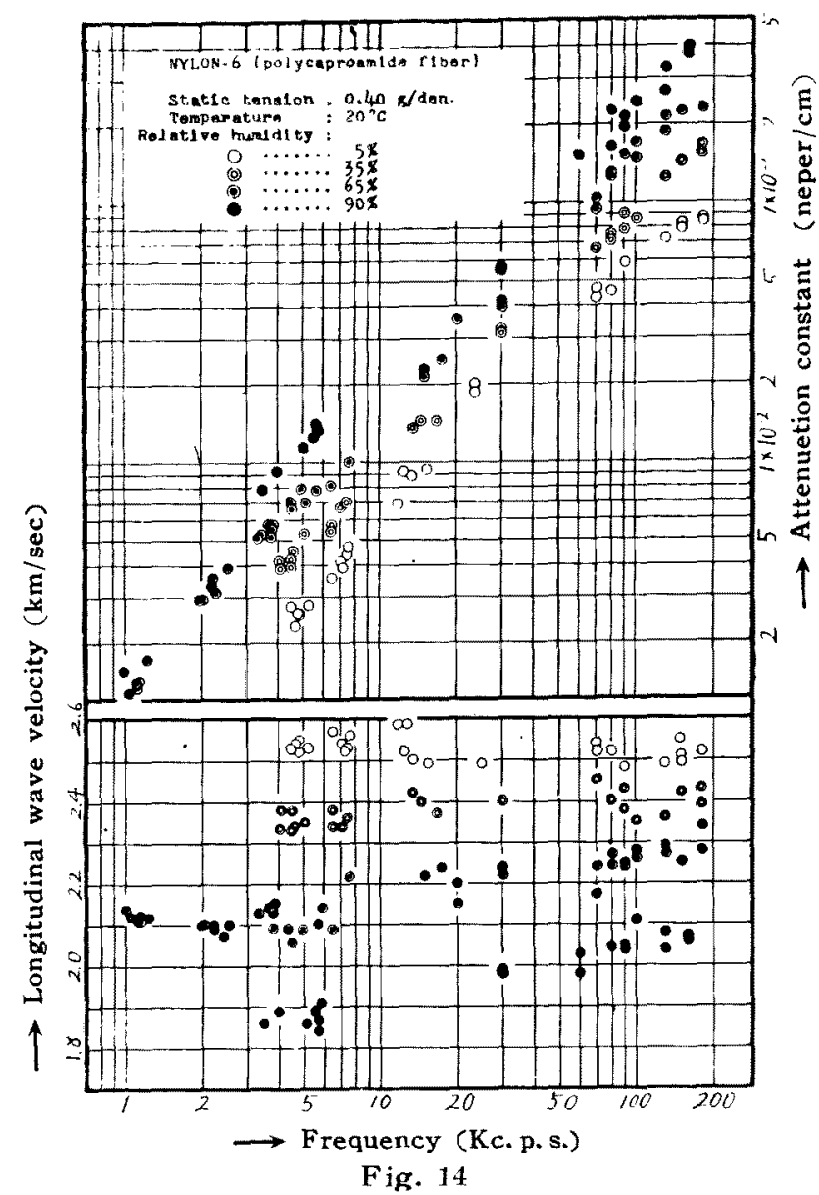

The respective values of tensile modulus of elasticity $E$ and of normal viscosity coefficient $\xi$ at a given angular frequency can be obtained by using Eqs. (12) and (13).

\section{Literature Cited}

(1) T. Alfrey and P. Doty, J. Appl. Phys., 16, 700 (1945); B. Gross, Mathematical structure of the theories of viscoelasticity, Paris, Hermann et Cie, (1953)

(2) K. Fujino, H. Kawai and T. Horino, Text. Res. J., 25. 722 (1955)

(3) R.S. Marvin, Ind. Eng. Chem., 44, 696 (1952)

(4) K. Fujino, H. Kawai and T. Horino, this journal, 1, 9 (1955)

(5) S. D. Gehman, D.E. Woodford and R.B. Stambaugh, Ind. Eng. Chem., 33, 1032 (1941)

(6) W. J. Lyons and I. B. Prettyman, J. Appl. Phys., 19, 473 (1947); Text. Res. J., 19, 123 (1949)

(7) B. A. Dunell and J.H. Dillon, Text. Res. J., 21, 393 (1951)

(8) G. Palandri, Rubber Age., 64, 45 (1948)

(9) A. W. Nolle, J. App. Phys., 19, 753 (1948)

(10) H. Kawai and N. Tokita, Report of the Kobayashi Institute of Phys. Res., 1, 265 (1951)

(11) N. Tokita, Journal of Japan Society for Testing Materials, 2, 14 (1953)

(12) J. W. Ballou and S. Silverman, J. Acoust. Soc. Am., 16, 113 (1944); Text. Res. J., 14, 282 (1944

(13) A. W. Nolle, J. Acoust. Soc. Am., 19, 194 (1948)

(14) J.W. Ballou and J.C. Smith, J. App. Phys., 20, 493 (1949)

(15) K. W. Hillier and H. Kolsky, Proc. Phys. Soc. Lond, B, 62, 111 (1949)

(16) K. W. Hillier, Proc. Phys. Soc. Lond., B, 62, 701 (1949)

(17) R.S. Witte, B.A. Mrowca and E. Guth, J. Appl. Phys., 22, 48 (11949 